\title{
The Effect of Oxytocin on Food Intake in the Nucleus Accumbens Core
}

\author{
Ying Zhao',2, Wang Qian¹, Wang Cheng ${ }^{1}$, Xu Luo1
}

\author{
${ }^{1}$ Qingdao University, Qingdao, Shandong, 266021, China \\ ${ }^{2}$ Heze Medical College, Qingdao, Heze, 274000, China
}

\begin{abstract}
Objective: In this experiment, we mainly investigated whether oxytocin (OT) acts on the Nucleus Accumbens Core (NAc) to regulate the feeding behavior of rats. Methods: Immunohistochemical experiment was used to observe the expression of OT receptor in NAc; To observe the effect of direct injection of OT in NAc on hunger- and palatability-driven food intake; c-Fos expression can be used as a marker of neuronal activation. After the NAc injection ofOT, the expression of c-fos in the hypothalamus was observed. Results: Studies found that the NAc injection of OT significantly reduced fasting-induced food intake and reduced intake of maltose and dextrin in non-fasted rats. Administration of the OTR antagonist L-368899 within NAc completely blocked these effects induced by OT. The results showed that intra-NAc injection of OT significantly activated NAc, as well as the other two brain regions associated with feeding: hypothalamic paraventricular nucleus and supraoptic nucleus. Conclusion: Injection of OT within NAc significantly attenuated starvation and reward-induced feeding behavior.
\end{abstract}

Keywords: Oxytocin; Arcuate nucleus; the Nucleus Accumbens Core; Food Intake

\section{Introduction}

In the 1950s, oxytocin (OT) was isolated in the pituitary gland and it was confirmed to be an amino acid sequence peptide with an active form. OT occurs in two areas of the hypothalamus, the supraoptic nucleus and paraventricular nucleus. The neurons that generate OT in the supraoptic nucleus are all large-cell neurons, and the paraventricular nucleus contains small-cell neurons in addition to large-cell neurons. Large cell neurons mainly act on the neurohypophysis. After OT synthesis, they are transported along the axons and stored in the neurohypophysis. When the body needs it, the neurohypophysis releases it into the blood circulation. The small cell neurons act on other parts of the brain, such as the brain stem tail, amygdala, substantia nigra, etc., mainly through the gastrointestinal vagal nerve reflex and other autonomous effects of the brain stem tail, regulating food intake.

In addition to playing an important role in the regulation of reproductive, digestive, stress, memory, neuroimmunity and other activities, OT has a close relationship with animal food intake and obesity ${ }^{[1]}$. OT promotes termination of feeding mainly through a central mechanism ${ }^{[1]}$. At the end of a meal, OT neuronal activity increases and OT release increases due to excessive plasma osmolality and excessive gastric stretch. There have been studies showed that rats fed the OT in the lateral ventricle were significantly reduced in food intake ${ }^{[2]}$. Subsequently, other studies have confirmed that OT has an anorexic effect that can lead to loss of appetite, and has begun to explore the role of OT in the brain parts of its anorectic effect. In addition, there are studies found that hypothalamic ventral nucleus $(\mathrm{VMH})$ injection of OT can significantly reduce the food intake in rats, and increase energy expenditure in rats ${ }^{[3]}$.

OT acts on brain regions outside the brain stem-hypothalamic pathway and can also lead to loss of appetite. It has been reported in the literature that VTA injection of OT on the ventral side of rats can significantly reduce food intake in fasted rats and reduce the intake of maltose solution in normal rats ${ }^{[4]}$. These findings are consistent with the results of other studies: the intake of carbohydrate and dextrin solution in OT knockout rats is significantly higher than that in normal rats. The OTR antagonist L-368899 was injected peripherally, and the intake of maltose and dextrin solution in fertilized mice was significantly increased, whereas intracerebroventricular injection of OT significantly reduced glucose intake in fasted rats. Injection of opioid peptide receptor antagonists can significantly reduce maltose uptake, mainly by activating OT neurons. OT gene expression was 
significantly increased in rats after a high-sugar diet ${ }^{[5]}$.

Central nervous system through the energy balance system and reward balance system to complete the regulation of the collective energy metabolism ${ }^{[1]}$. The nucleus accumbens (NAc) is the main part of the ventral striatum, which is divided into the shell and the nucleus and is the core structure of the reward balance system. Studies have shown that studies on the relationship between NAc and feeding have shown that intranuclear injection of opioid peptide receptor antagonists and agonists, as well as dopamine receptor antagonists can affect rats feeding. Opiate and dopamine signaling in NAc can affect feeding preferences, and glutamate and GABA may be involved in regulating energy homeostasis. Studies have also shown that GLP-1 in NAc may affect food intake and food reward ${ }^{[6]}$. Although OTR is mainly distributed in $\mathrm{NAc}^{[2]}$, no study has reported this brain region is involved in regulating the effects of OT on feeding.

In this experiment, we mainly observed that the expression of OT receptor in NAc and whether OT acted on NAc affected the feeding behavior of rats. We observed whether intra-NAc injection of OT affects starvation, sweets, and delicious food-induced rat food intake.

\section{Experimental materials and methods Animals}

Adult male SD rats, weighing $300 \mathrm{~g}$. All rats were housed in room temperature is $25 \pm 2^{\circ} \mathrm{C}$ and $12 \mathrm{~h}: 12 \mathrm{~h}$ of day and night cycling light, given a standard laboratory diet, free access to food and drinking water. All animal experiments were strictly conducted according to the "Qingdao University Laboratory Animal Protection and Use Management Measures."

\section{Immunohistochemistry Experiment}

Rats were anesthetized with sodium pentobarbital (50 $\mathrm{mg} / \mathrm{kg}$, ip) and fixed on the operating table, Then injection of $250 \mathrm{ml} 0.9 \%$ saline and $250 \mathrm{ml} 4 \%$ paraformaldehyde for perfusion fixation. The rats were decapitated and their brains were removed. The slices were continuously sectioned using a cryostat and the thickness was $15 \mu \mathrm{m}$. All slices were stored in a refrigerator at $-20^{\circ} \mathrm{C}$ to avoid light and frozen. The NAc sections were selected for immunofluorescence staining of NPY5 receptors and then the experimental results were observed under a fluorescence microscope.

\section{Brain cannula implantation}

Rats were anesthetized with sodium pentobarbital (50 $\mathrm{mg} / \mathrm{kg}$, i.p) and fixed in a stereotaxic apparatus. A stainless steel was implanted into NAc (front anterior: $2.2 \mathrm{~mm}$; lateral: $1.5 \mathrm{~mm}$; subcranial: $7.0 \mathrm{~mm}$ ) according to the Paxinos \& Watson rat brain map.
Postoperative injections of antibiotics prevent infection. Rats can resume follow-up experiments after at least 7 days of recovery.

In order to detect whether the positioning is accurate, administration indole sky blue solution in the ARC $\mathrm{h}$ at the end of the experiment. the rat is perfused through the heart, and the brain is rapidly decapitated. A $50 \mu \mathrm{m}$ coronal frozen section was made and the position of the drug injection was observed under a microscope. Experimental data where the injection site is not in the $\mathrm{ARC}$ is not used in this experiment.

\section{Drug administration}

The OT and OT receptor antagonists L-368899 were all dissolved in isotonic saline and injected into NAc through the brain nucleus. The time interval between the injection of the drug and the administration of the food or the time interval between the two injections of the drug during the experiment is calculated from the time that the first injection of the drug is completed.

\section{The effect of NAc administration of OT on Food Intake in Rats}

In the experiment, 30 rats were randomly divided into 6 groups (n=5): (1) saline group; (2) $0.3 \mu \mathrm{g}$ OT group; (3) $1.0 \mu \mathrm{g}$ OT group; (4) $3.0 \mu \mathrm{g}$ OT group. The rats were fasted overnight and the corresponding doses of drugs were injected into the NAc separately. Rats were given foods that had been previously weighed and the remaining amount of food was measured at $2 \mathrm{~h}$ and $4 \mathrm{~h}$ after injection of the corresponding drugs in each group of rats.

\section{The effect of NAc administration of OT on maltose solution and dextrin solution intake}

This experiment refers to the previous research method ${ }^{[7]} .30$ rats were randomly divided into 6 groups $(n=5)$ : NS group, $0.1 \mu \mathrm{g}$ OT group, $0.3 \mu \mathrm{g}$ OT group, $1.0 \mu \mathrm{g}$ OT group, and $3.0 \mu$ g OT group. Rats were given a $10 \%$ maltose solution every day for a fixed period of time (10:00-12:00) for a total of 3 days (rats removed food and water during the intake of maltose).On the 4th day, the NAc was injected with the corresponding drugs, and 5 min was given to the rat $10 \%$ maltose solution. After each group of rats was injected with the corresponding drug, the maltose solution intake was measured for 2 hours. The same method was used to observe the effect of NAc injection OT on the intake of $0.1 \%$ dextrin. The difference is that the OT dose used was $0.01 \mu \mathrm{g}, 0.03 \mu \mathrm{g}, 0.1 \mu \mathrm{g}, 0.3 \mu \mathrm{g}$, and $3.0 \mu \mathrm{g}$, respectively.

\section{Effect of intranasal injection of OTR antagonists on food intake in fasted rats}

Twenty-five rats were randomly divided into 6 groups with 5 in each group: (1) NS+NS group: First 
intra-NAc injection of NS; 10 min after injection of NS; (2) NS+1.0 $\mu$ g OT group : First injection of NS in NAc, $1.0 \mu \mathrm{g}$ OT after $10 \mathrm{~min}$; (3) $0.3 \mu \mathrm{g} \mathrm{L-368899}+1.0 \mu \mathrm{g}$ OT group: $0.3 \mu \mathrm{g}$ L-368899 within the first NAc injection, $1.0 \mu \mathrm{g}$ OT after $10 \mathrm{~min}$ (4) $1.0 \mu \mathrm{g}$ L-368899 $+1.0 \mu \mathrm{g}$ OT group: $1.0 \mu \mathrm{g} \mathrm{L-368899}$ within the first NAc injection, $1.0 \mu \mathrm{g}$ OT after $10 \mathrm{~min}$; (5) $3.0 \mu \mathrm{g}$ L-368899 + $1.0 \mu \mathrm{g}$ OT group: One injection of $3.0 \mu \mathrm{g}$ L-368899 within one NAc and $1.0 \mu \mathrm{g}$ OT after 10 minutes. The rats were fasted overnight and each group of rats was injected with the corresponding drugs and given foods that had been previously weighed. The food intake was measured at $2 \mathrm{~h}$ and $4 \mathrm{~h}$ in each group of rats.

\section{Effects of Intracellular NAc Injection of OTR Antagonists on the Intake of Maltose, Solution and Dextrin Solution}

The experiment was divided into two parts: Part I: To observed the effect of pre-injection of OTR antagonists on the intake of maltose solution in NAc.In the experiment, 15 rats were randomly divided into 3 groups with 5 animals in each group: (1) NS + NS group: the first injection of NS in NAc; 10 min later injection of NS; (2) NS + $0.3 \mu \mathrm{g}$ OT Group: First injection of NS in NAc, $0.3 \mu \mathrm{g}$ OT after $10 \mathrm{~min}$; (3) 1.0 $\mu \mathrm{g}$ L-368899 + $0.3 \mu \mathrm{g}$ OT group: $1.0 \mu \mathrm{g}$ L-368899 within the first NAc injection, $0.3 \mu \mathrm{g}$ after $10 \mathrm{~min}$ OT. Rats were given a $10 \%$ maltose solution every day for a fixed period of time (10:00-12:00) for a total of 3 days (rats removed food and water during the intake of maltose).On the 4th day, rats were injected with the corresponding drug in NAc respectively. After $5 \mathrm{~min}$, $10 \%$ maltose solution was given to the rats and the maltose solution intake was measured for $2 \mathrm{~h}$.

Part II: To observe the effect of pre-injection of OTR antagonists on the intake of $0.1 \%$ dextrin solution in NAc.In the experiment, 15 rats were randomly divided into 3 groups with 5 rats in each group: (1) NS + NS group: first injection of NS in NAc; 10 minutes later, NS injection again; (2) NS+0.03 $\mu \mathrm{g}$ OT Group: First injection of NS in NAc, $0.03 \mu \mathrm{g}$ OT after $10 \mathrm{~min}$; (3) $3.0 \mu \mathrm{g}$ L-368899 + $0.03 \mu \mathrm{g}$ OT group: $3.0 \mu \mathrm{g}$ L-368899 within the first NAc injection, $0.03 \mu \mathrm{g}$ after $10 \mathrm{~min}$ OT. Rats were given a $0.1 \%$ dextrin solution every day for a fixed period of time (10:00-12:00) for a total of 3 days (rats removed food and water during dextrin intake).On the 4th day, rat NAc was injected with the corresponding drug, respectively, and 5 min later was given to the rat $0.1 \%$ dextrin solution, and the $2 \mathrm{~h}$ dextrin solution intake was measured.

\section{C-fos expression}

10 rats were randomly divided into 2 groups: saline group $(n=5)$ and OT group $(n=5)$. The saline and OT $(3.0 \mu \mathrm{g})$ were injected intranasally into the NAc, respectively, and the drug injection time was between 10:00-12:00.Rats banned feeding and drinking after drug injection. After $60 \mathrm{~min}$, rats were anesthetized with sodium pentobarbital (50 $\mathrm{mg} \mathrm{/} \mathrm{kg})$ by intraperitoneal injection, fixed on the operating table, fixed in saline and $4 \%$ paraformaldehyde, and rapidly removed from the rat brain. After placing in $4 \%$ paraformaldehyde for 24 hours, the rat brain was placed in $0.1 \mathrm{M}$ PBS and rinsed three times for 8 hours each. The rinsed brain tissue was stored in $75 \%$ alcohol.Select suitable brain tissue for staining.Brain tissue sections were incubated overnight at $4{ }^{\circ} \mathrm{C}$ with c-fos antibody, then placed in biotinylated goat anti-rabbit $\operatorname{IgG}$ for $2 \mathrm{~h}$, and placed in avidin-biotin-peroxidase complex for $1 \mathrm{~h}$. The brain slices were fully reacted with $0.02 \% 3,3-\mathrm{DAB}$ solution, $0.3 \%$ nickel sulfate, and $0.005 \%$ hydrogen peroxide to give a brown-yellow precipitate.The stained sections were placed in $90 \%$ ethanol and dehydrated for 10-20 s, and then placed in different concentrations of ethanol solution for dehydration. After dehydration, the sections were xylene transparent 3 times, each 2-3 min. The transparent sections were sealed with a neutral gum and the results were observed under a microscope and photographed.SCION IMAGE software was used to evaluate the number of c-fos immunopositive neurons in the corresponding brain regions.

\section{Statistical analysis}

The experimental data were expressed as mean \pm SD. The experimental data were statistically analyzed with Prism 5.0 software. T test or one-way ANOVA was used between the two groups. $\mathrm{P}<0.05$ was considered statistically significant.

\section{Results}

\section{Expression of OT receptors in Nac}

Immunofluorescence histochemistry results showed that OT receptor expression was observed in NAc. 

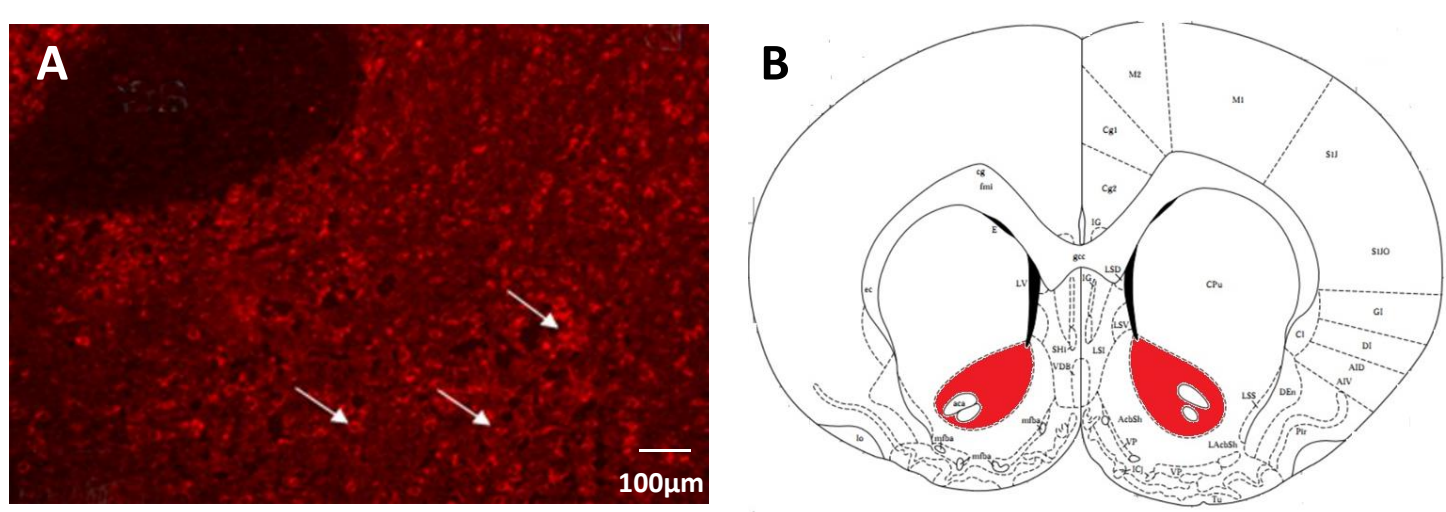

Fig.1 Expression of OT receptors in NAc.

A: Expression of OT receptors in NAc; B: Schematic drawing illustrating the position in the NAc

\section{Effect of intranasal injection of OT on food intake} in fasted rats

The experimental results show that direct injection of OT within NAc can affect food intake in fasted rats (Fig. 2). OT $1.0 \mu \mathrm{g}$ and $3.0 \mu \mathrm{g}$ OT injections within NAc significantly reduced the cumulative food intake in fasted rats $0-2 \mathrm{~h}$ and $0-4 \mathrm{~h}(\mathrm{P}<0.05-0.01$, Fig. 2). There was no significant change in water intake at $4 \mathrm{~h}$ after injection (Fig. 3).

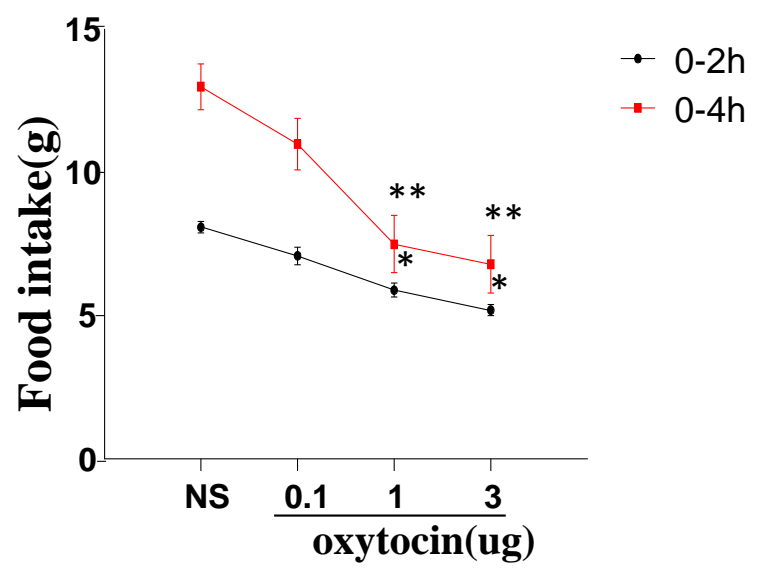

Fig.2Effects of different doses of oxytocin (OT) injected into theNAc on food intake of 0-2hand 0-4hin deprivation rats.

$* \mathrm{P}<0.05, * * \mathrm{P}<0.01$, compared with the NS group.

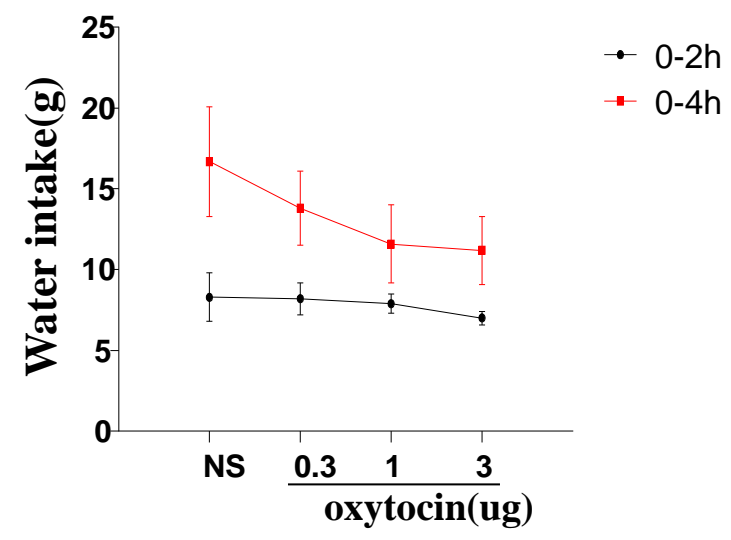

Fig.3 Effects of different doses of oxytocin (OT) injected into the NAc on water intake of $0-2 \mathrm{~h}(\mathrm{~A})$ and $0-4 \mathrm{~h}(\mathrm{~B})$ in deprivation rats.

NAc injection OT on the maltose solution and dextrin solution intake

The experimental results showed that the injection of $1.0 \mu \mathrm{g}$ and $3.0 \mu \mathrm{g}$ of OT in NAc significantly decreased the intake of maltose solution, with a $50 \%$ reduction $(\mathrm{P}<0.05$, Fig. 4). Injections of $0.03 \mu \mathrm{g}, 0.1 \mu \mathrm{g}, 0.3 \mu \mathrm{g}$, and $1.0 \mu \mathrm{g}$ of OT in NAc reduced dextrin solution intake, but required lower OT doses. 

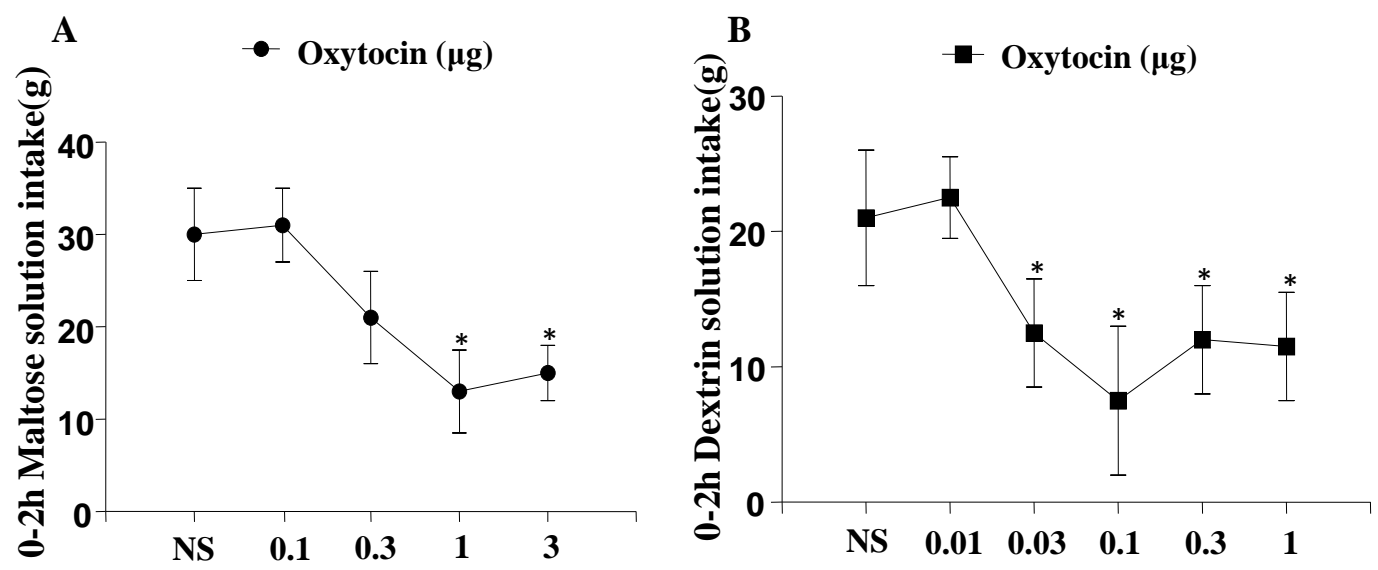

Fig.4 Effects of different doses of oxytocin (OT) injected into the NAc on the intake of $10 \%$ maltose solution (A) and $0.1 \%$ dextrin(B) solution during the $0-2 \mathrm{~h}$ in rats.

$* \mathrm{P}<0.05$, compared with the NS group

\section{NAc Injected OTR Antagonist Influences} OT-induced Anorexia Effect

NAc pre-injection of OTR antagonists eliminates the anorectic effect induced by OT. In fasted rats, pre-injection of $3.0 \mu \mathrm{g}$ of L-368,899 can offset OT for
0-2 h $(\mathrm{P}<0.05$, Fig. 5) compared with saline + saline, NAc pre-injection of $1.0 \mu \mathrm{g}, \quad 3.0 \mu \mathrm{g} \quad \mathrm{L}-368899$ eliminated the anorexia induced by OT in $0-4 \mathrm{~h}$ $(\mathrm{P}<0.01$, Fig. 5).

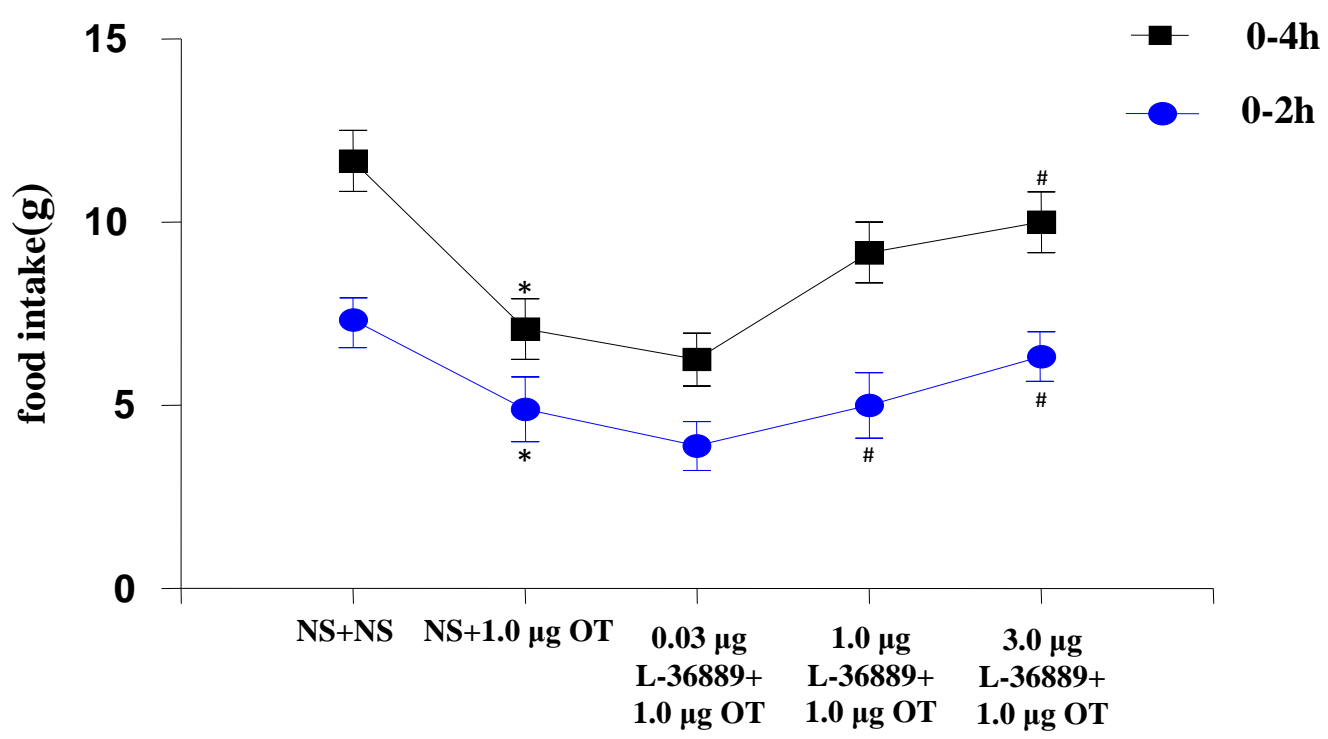

Fig.5 Effect of pre-injection of oxytocin (OT) receptor antagonist (L-368899) on OT-induced anorexia effects in nucleus accumbens (NAc).

$* \mathrm{P}<0.05$, compared with the NS+NS group; $\# \mathrm{P}<0.05$, compared with the $\mathrm{NS}+1.0 \mu \mathrm{g}$ OT group

The experiment also found that the OTR antagonist L-368899 could also block the inhibitory effect of OT on rat $10 \%$ maltose solution and $0.1 \%$ dextrin solution ingestion compared with saline + saline $(\mathrm{P}<0.05$, Fig. 6). 


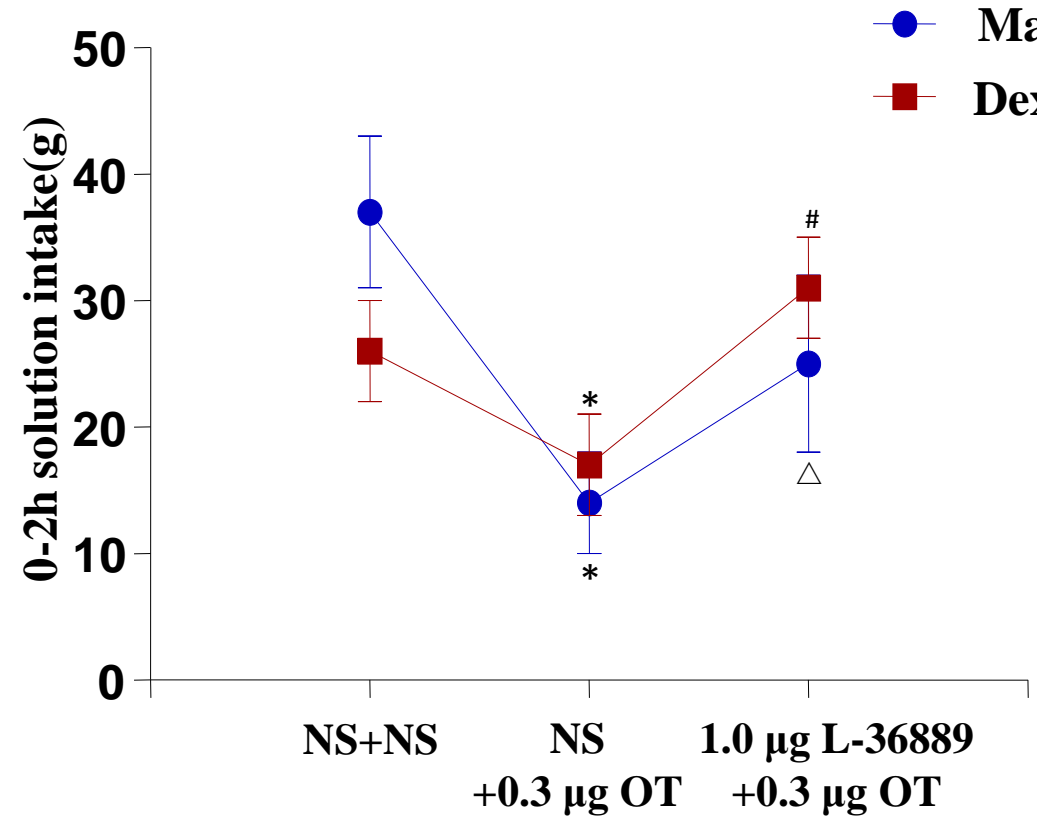

Fig.6Effect of pretreatment with oxytocin (OT) receptor antagonist (L-368899) on OT-induced maltose solution and dextrin solution intake in nucleus accumbens (NAc).

${ }^{*} \mathrm{P}<0.05$, compared with the NS+NS group; \#P<0.05, compared with the NS $+0.3 \mu$ g OT group; $\triangle \mathrm{P}<0.05$, compared with the NS $+0.03 \mu \mathrm{g}$ OT group

\section{C-fos expression}

The results of immunohistochemistry showed that compared with the saline group, NAc, PVN, SON and other parts showed a significant increase in c-fos expression after NAc injection of $3.0 \mu \mathrm{g}$ OT, indicating that NAc injection can not only activate NAc itself, but also activate it. PVN, SON, and other brain areas associated with feeding.

Table1The expression of c-Fos in feeding-related brain sites after the nucleus accumbens core (NAc) administration

\begin{tabular}{cccccccc}
\hline \multicolumn{8}{c}{ of oxytocin $(\mathrm{OT})$ or NS } \\
\hline Groups & NAc & PVN & SON & DMH & LHA & ARC & VTA \\
\hline NS & $124.1 \pm 24.2$ & $131.3 \pm 18.8$ & $71.9 \pm 28.4$ & $131.3 \pm 24.9$ & $68.8 \pm 15.6$ & $106.3 \pm 19.1$ & $87.7 \pm 9.6$ \\
OT & $275.2 \pm 24.5$ & $237.5 \pm 18.6$ & $181.3 \pm 37.5$ & $112.6 \pm 24.8$ & $75.4 \pm 19.1$ & $87.6 \pm 31.7$ & $100.4 \pm 18.8$ \\
\hline
\end{tabular}



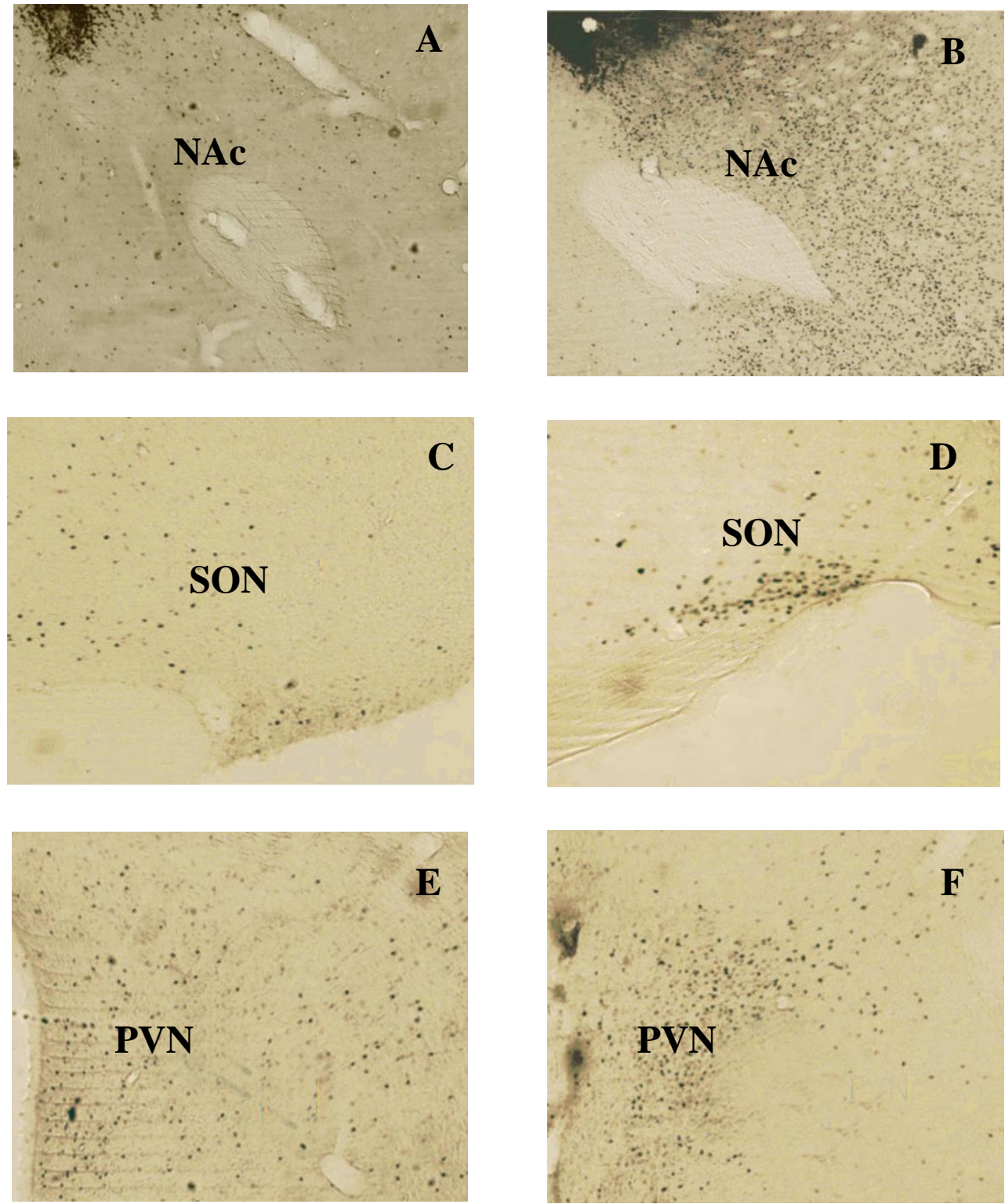

Fig.7 The expression of c-Fos in feeding-related brain sites after the nucleus accumbens core (NAc) administration of oxytocin (OT) or NS.

A: The expression of C-fos in NAc after NAc injection of NS; B: The expression of C-fos in NAc after NAc injection of OT; $\mathrm{C}$ : The expression of $\mathrm{C}$-fos in NAc after PVN injection of NS; D: The expression of C-fos in NAc after PVN injection of OT; E: The expression of $\mathrm{C}$-fos in NAc after SON injection of NS; F: The expression of C-fos in NAc after SPN injection of OT. NAc: the nucleus accumbens core; SON: supraoptic nucleus; PVN: paraventricular hypothalamic nucleus.

\section{Discussion}

The central endogenous OT participates in the inhibition of feeding and has the effects of producing satiety, inhibiting gastric motility, and feeding. Recent studies have shown that OT can reduce the intake of carbohydrates and delicious foods by acting on neural networks including VTAs. The function of OT involves a broader neural network including VTA, which reduces the intake of carbohydrates and delicious sweeteners, and for the first time identified NAc as an important site for OT to participate in the regulation of energy balance.

This experiment found that direct injection of OT in NAc can reduce the starvation-induced feeding behavior, but also reduce the intake of delicious sweeteners. Pre-OTR antagonist L-368, 899 in NAc can reverse the effect of OT, the experimental results show that within NAc, OTR may be involved in the regulation of OT-induced feeding changes. The above 
studies show that although the hindbrain OTR plays a key role in regulating energy balance, this receptor in the forebrain NAc should also be considered as an important mechanism for central regulation of calorie intake.

An important study of OT's involvement in regulating appetite focuses on the effects of OT on the intake of carbohydrates and delicious sweeteners. OT knockout mice exhibit increased carbohydrate and saccharin intake $^{[7]}$. The wild-type mice were injected peripherally with the OTR antagonist L-368,899 (permeable to the blood-brain barrier), which promoted the intake of monosaccharides, disaccharides, polysaccharides, and saccharin in mice. The results of this study confirm that OT plays a key role in reward-reward activities. This study shows that microinjection of OT in NAc can reduce the consumption of sweetener solutions (maltose and dextrin) at a dose equal to or lower than that of $\mathrm{VTA}^{[4]}$.

In addition, intranasal injection of OT induces increased expression of c-Fos in PVN and SON, similar to the activation of the relevant neurons following food intake, including palatable or common food and reward-driven eating behavior ${ }^{[8]}$. Since the endogenous pathway of the hypothalamus can locally modulate the activity of PVN, it was observed in this study that the increase in c-Fos expression in the hypothalamus may also result from the activation of neurons within a wider range of neural networks. Existing studies have also observed changes in feeding caused by OT injection within NAc, which is similar to the effect of injection of GLP-1 into NAc ${ }^{[6]}$. The role of GLP-1 in NAc is similar to that of OT, and both can participate in the regulation of food intake and reward systems.

In conclusion, this study found that intra-NAc injection of OT reduced starvation-induced feeding behavior and reduced rat intake of delicious foods and sweeteners. Injection of OT in NAc affects the activity of NAc itself, and may also affect the hypothalamus involved in the regulation of feeding.

\section{References}

1. Feng Z, Ou Y, Zhou M, et al. Functional ectopic neural lobe increases GAP-43 expression via PI3K/AKT pathways to alleviate central diabetes insipidus after pituitary stalk lesion in rats[J]. NeurosciLett, 2018, 673(8):1-6

2. Maejima Y, Takahashi S, Takasu K, et al. Orexin action on oxytocin neurons in the paraventricular nucleus of the hypothalamus[J]. Neuroreport, 2017, 12, 28(6):360-366

3. Mandal A, Prabhavalkar KS, Bhatt LK. Gastrointestinal hormones in regulation of memory[J]. Peptides, 2018, 18(10):16-25

4. Mullis K, Kay K, Williams DL. Oxytocin action in the ventral tegmentalarea affects sucrose intake[J]. Brain Res, 2013, 1513(8): 85-91
5. Olszewski PK, Shaw TJ, Grace MK, et al. Complexity of neural mechanisms underlying overconsumption of sugar in scheduled feeding: involvement of opioids, orexin,oxytocin and NPY[J]. Peptides, 2009, 30(4): 226-233

6. Samplaski MK, Lo KC, Grober ED, et al. Varicocelectomy to "upgrade" semen quality to allow couples to use less invasive forms of assisted reproductive technology[J]. FertilSteril, 2017, 108(4):609-612

7. Liu W, Zhang C, Zhang Y, et al. Effects of electroacupuncture at acupoints with different nerve segments on gastric emptying in OT gene knockout mice[J]. Zhongguo Zhen Jiu, 2017 , 37(7):735-740

8. Hankir MK, Patt M, Patt JT, et al. Suppressed Fat Appetite after Roux-en-Y Gastric Bypass Surgery Associates with Reduced Brain $\mu$-opioid Receptor Availability in Diet-Induced Obese Male Rats[J]. Front Neurosci, 2017, 10(3):620 